\title{
Molecular Dynamics Simulation of Composite Nanochannels as Nanopumps Driven by Symmetric Temperature Gradients
}

\author{
Chong Liu and Zhigang $\mathrm{Li}^{*}$ \\ Department of Mechanical Engineering, The Hong Kong University of Science and Technology, \\ Clear Water Bay, Kowloon, Hong Kong \\ (Received 10 May 2010; revised manuscript received 28 June 2010; published 18 October 2010)
}

\begin{abstract}
In this Letter, we propose a composite nanochannel system, where half of the channel is of low surface energy, while the other half has a relatively high surface energy. Molecular dynamics simulations show that fluids in such channels can be continuously driven by a symmetric temperature gradient. In the low surface energy part, the fluid moves from high to low temperature, while the fluid migrates from low to high temperature in the high surface energy part. The mechanisms that govern the flow are explained and the conditions required to guarantee the flow and the possible applications are discussed.
\end{abstract}

DOI: 10.1103/PhysRevLett.105.174501

PACS numbers: $47.61 .-\mathrm{k}$

Liquid transport in micro- or nanochannels is of great importance in many applications, including biomolecule separation, energy conversion, and thermal management [1-4]. Mechanical, electrokinetic, and acoustic approaches can be used to drive the fluid through the channel [4-8]. The fluid can also be circulated by heterogeneous forces caused by a surface tension, chemical, or temperature gradient [1,9-11]. However, in certain applications, these gradients can be symmetric and the total net force on the fluid vanishes. In this case, the fluid cannot be constantly transported without external forces. Inspired by our recent work on the flow regimes in nanochannels [12], here we propose a composite nanochannel system, where half of the channel has low and the other half has relatively high surface energy, as will be explained later. Through molecular dynamics simulations, it is shown that liquids in such channels can be continuously pumped by a symmetric temperature gradient along the channel. One advantage of this system is the application for chip-level cooling, where the heat generated in the chip can be used to drive the liquid without using external pumps, which consume energy, occupy space, and therefore conflict with the miniaturization objectives of next generation electronic devices.

The composite nanochannel system is illustrated in Fig. 1. The channel is formed by two parallel walls. A liquid (green particles), which is in connection with two reservoirs, is confined by the walls. For the convenience of numerical simulation, the same structure and potential are used for the walls. The composite channel is defined in terms of the surface energy or fluid-wall interaction, which is heterogeneous and realized by controlling the fluid-wall binding energy $\varepsilon_{\mathrm{fw}}$. The binding energy between the fluid and left half of the wall (orange) $\varepsilon_{\mathrm{fw}(L)}$ is weak (low surface energy) and that between the fluid and right half of the wall (gray) $\varepsilon_{\mathrm{fw}(R)}$ is strong (high surface energy). The low or high surface energy represents different types of molecular interactions. For low surface energy, the fluid-wall interaction is weak and mainly repulsive, while both repulsive and attractive interactions are strong for high surface energy.

The channel walls are constructed by truncating a rectangular portion from a face-centered cubic structure with a lattice constant equal to $4.086 \AA$. There are four layers of atoms in each wall. The atoms in the outmost layers are fixed to maintain a stable system whereas the atoms in the other three layers are free to vibrate to consider the flexibility of the wall. The lengths of the simulation cell in the $x$ and $z$ directions are 16 and $5 \mathrm{~nm}$, respectively. The length of the wall in the $x$ direction is $8 \mathrm{~nm}$. The channel width, distance between the innermost atomic layers in the $y$ direction, is varied from 2 to $6 \mathrm{~nm}$. The wall is modeled by the tight-binding potential, which has been well accepted for transition metals, and the parameters for $\mathrm{Ag}$ are used [12]. (Note that the wall can simply be modeled by confining the atoms to their lattice sites with a restoring force [13]. The potential may affect the fluid dynamical properties near the fluid-wall interface, such as the velocity slip length.) The fluid-fluid interaction is modeled by the Lennard-Jones 12-6 potential, $U(r)=4 \varepsilon_{\mathrm{ff}}\left[\left(\sigma_{\mathrm{ff}} / r\right)^{12}-\right.$ $\left.\left(\sigma_{\mathrm{ff}} / r\right)^{6}\right]$, where $r$ is the intermolecular distance, $\varepsilon_{\mathrm{ff}}$ is

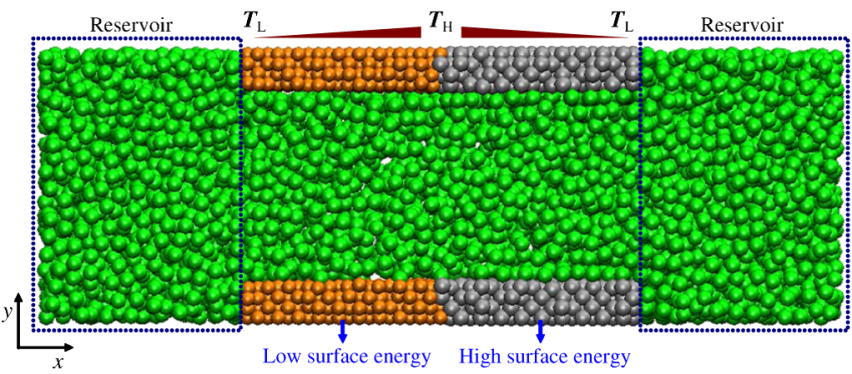

FIG. 1 (color online). Structure of the composite nanochannel system under a symmetric temperature gradient. Green particles are liquid molecules, which are in connection with two reservoirs. The left half of the wall (orange) has low and the right half (gray) has high surface energy. The temperature in the middle is higher than that at the ends of the channel. 
the fluid-fluid binding energy, and $\sigma_{\mathrm{ff}}$ is the collision diameter of the fluid molecules. The values of $\varepsilon_{\mathrm{ff}}$ and $\sigma_{\mathrm{ff}}$ are set to be $9.8 \times 10^{-3} \mathrm{eV}$ and $3.47 \AA$ and the fluid number density $\rho_{0}=15.381 / \mathrm{nm}^{3}$. The fluid-wall interaction is also calculated by the Lennard-Jones potential. To control the surface energy and understand the effect of surface energy heterogeneity on the flow, the fluid-wall binding energy $\varepsilon_{\mathrm{fw}}$ is arbitrarily varied. Temperature gradients are imposed on the wall such that the temperature around the middle $T_{H}$ is higher than that around the ends of the wall $T_{L} . T_{H}$ and $T_{L}$ are maintained at desired values by controlling the kinetic energy of wall atoms lying in the slit of $5 \AA$ around the midline and ends of the walls through the Berendsen thermostat [14]. Periodic boundary conditions are employed in the $x$ and $z$ directions only. The potentials are truncated at $10.21 \AA$ and Newton's equations are integrated with Beeman's leapfrog algorithm [15], with the time step equal to $1 \mathrm{fs}$. The system is relaxed for up to $2 \mathrm{~ns}$ to reach steady state before the flow properties are computed.

We first investigate the volumetric flux of the liquid in $4 \mathrm{~nm}$ channels under different temperature gradients and surface conditions. The temperature at the ends of the channel $T_{L}$ is maintained at $100 \mathrm{~K}$ and that in the middle $T_{H}$ is changed from 300 to $750 \mathrm{~K}$. The binding energy between the fluid and right half of the channel, which has high surface energy, is fixed at $\varepsilon_{\mathrm{fw}(R)}=500 \mathrm{~K}$ and that between the fluid and left half of the channel $\varepsilon_{\mathrm{fw}(L)}$ is varied from 10 to $200 \mathrm{~K}$ to consider the effect of surface energy configuration on the flow rate. Figure 2 shows the volumetric flux of the liquid as a function of $\varepsilon_{\mathrm{fw}(L)}$ under different temperature gradients. The reduced flux in Fig. 2 is the flux scaled by $\sqrt{\mathrm{eV} / m}$, where $\mathrm{eV}$ is electron volt and $m$ is the mass of a fluid molecule. It is seen that the

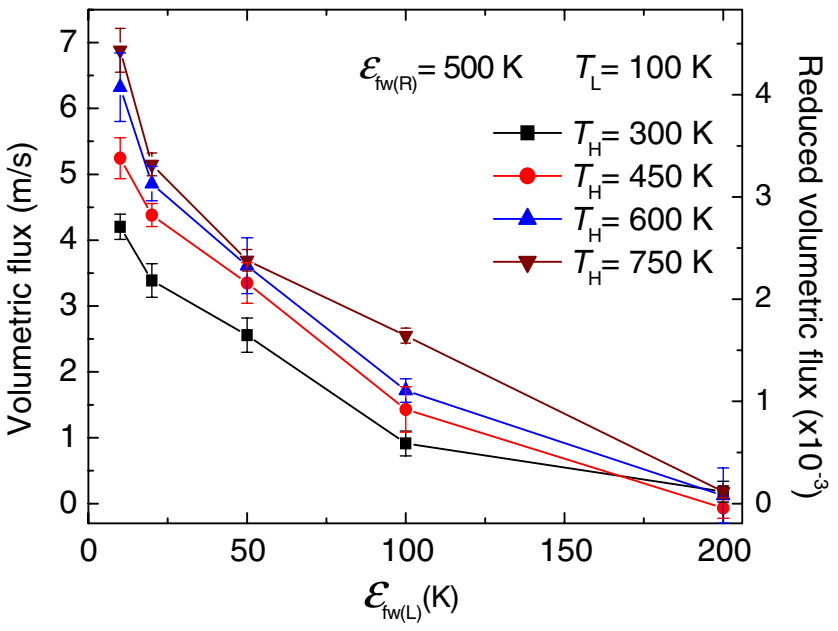

FIG. 2 (color online). The volumetric flux of the liquid as a function of $\varepsilon_{\mathrm{fw}(L)}$ at different temperature gradients in a $4 \mathrm{~nm}$ channel. The fluid-wall binding energy in the right half of the channel $\varepsilon_{\mathrm{fw}(R)}=500 \mathrm{~K}$ and $T_{L}=100 \mathrm{~K}$. fluid does have a clear unidirectional motion under the heterogeneous surface energies and symmetric temperature gradient. The flux increases with increasing surface energy heterogeneity and high temperature gradients generally enhance the flow rate.

The mechanisms that the composite channel can serve as a nanopump are nontrivial. The flow in the channel is determined by different fluid-wall interaction scenarios, which lead to nonuniform density and pressure distributions under the temperature gradient. The fluid pressure $P$ can be estimated through the virial equation of state [14]

$$
P=\rho k T+\sum_{i} \sum_{j>i} r_{i j} F_{i j} / 3 V,
$$

where $\rho$ is the number density, $k$ is the Boltzmann constant, $F$ is the force between a pair of molecules, and $V$ is the volume. In Eq. (1), the two terms on the right-hand side are the kinetic and potential components of the pressure, respectively. The contributions of these two components to the pressure variation are different in the low and high surface energy parts of the channel.

In the low surface energy part (left half), the binding energy $\varepsilon_{\mathrm{fw}(L)}$ is relatively weak and the fluid-wall interaction is mainly in the form of elastic collisions without fluid adsorption [12]. The role of temperature lies in affecting the kinetic energy and pressure of the fluid. At high temperature (middle of the channel), the kinetic energy and therefore the pressure of the fluid are higher than those at low temperature. Hence, the fluid in the left half of the channel moves from the middle (high temperature) to the left end (low temperature) of the channel, as will be confirmed by the velocity field later. Note that the temperature gradient can induce a density gradient, which may weaken the pressure variation. However, the density change is insignificant compared with the temperature gradient, as shown in Figs. 3(a) and 3(b). Therefore, in this part, the pressure gradient, based on Eq. (1), is mainly caused by the kinetic component due to the temperature gradient (this is confirmed by the pressure distributions due to the two parts in Eq. (1), which are not reported here).

In the high surface energy part (right half), however, the fluid-wall binding energy is strong, which causes fluid adsorption on the wall [12]. The number of fluid molecules adsorbed by the wall is affected by the temperature variation in the wall. Low temperature favors fluid adsorption because the kinetic energy of the fluid molecules is insufficient to overcome the wall attraction. Therefore, the strong fluid-wall interaction and temperature gradient generate a large density gradient in the fluid, as shown in Fig. 3(a), and the highest density takes place around the wall at the low temperature end. The nonuniform density distribution leads to heterogeneous intermolecular interactions and pressure variation [Fig. 3(c)]. The fluid-fluid repulsive force is stronger in high density area than that in low density area, which will cause the fluid molecules to migrate from high to low density regions. Suppose the 

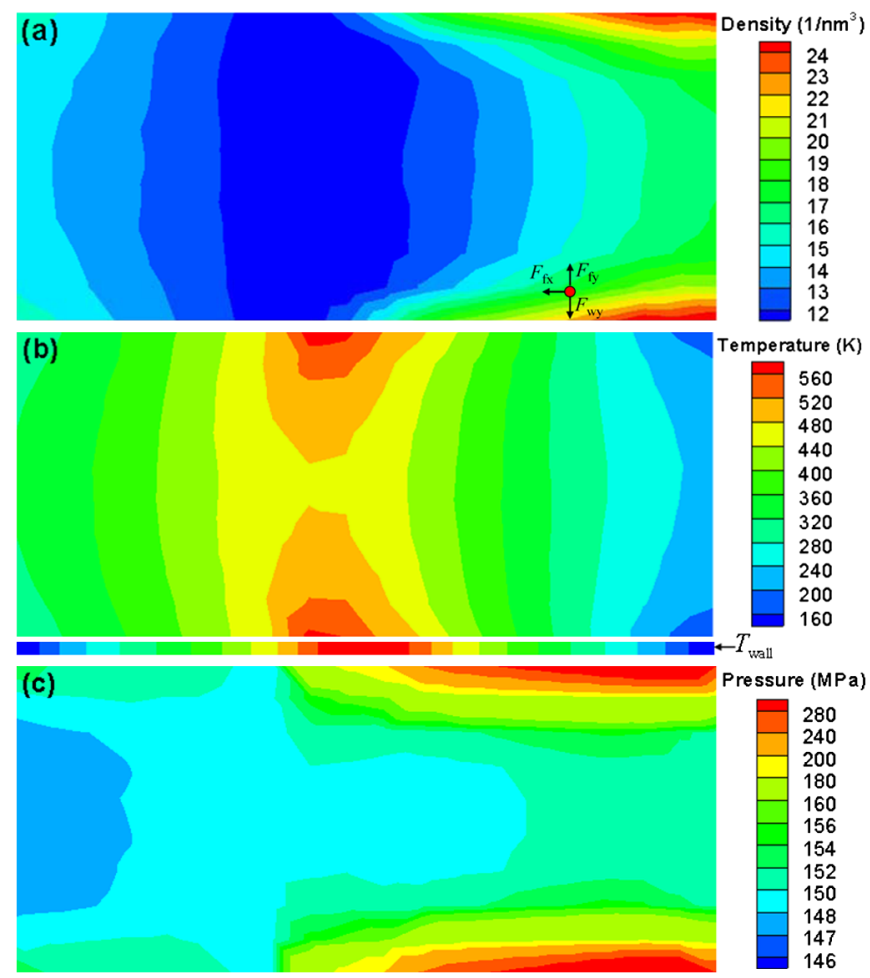

FIG. 3 (color online). Two-dimensional contour plots of (a) density, (b) temperature, and (c) pressure distribution in the channel for the flow in a $4 \mathrm{~nm}$ channel with $T_{H}=600$ and $T_{L}=100 \mathrm{~K}$. The fluid-wall binding energy $\varepsilon_{\mathrm{fw}(L)}=10$ and $\varepsilon_{\mathrm{fw}(R)}=500 \mathrm{~K}$. The thin color strip in (b) shows the temperature in the wall.

intermolecular forces can be represented by two forces in the $x$ and $y$ directions, $F_{\mathrm{fx}}$ and $F_{\mathrm{fy}}$, any fluid molecule will generally move to the left because the attractive force from the wall $F_{\mathrm{wy}}$ is in the $y$ direction and $F_{\mathrm{fx}}$ cannot be balanced, as shown in Fig. 3(a). The magnitudes of $F_{\text {fy }}$ and $F_{\text {wy }}$ determine whether the molecule will move toward the center or wall, as will be shown in the velocity field. In this part, the potential component of the pressure due to the large density variation dominates over the kinetic component. Therefore, in the right half, the fluid moves from low to high temperature and the pressure difference is mainly caused by the density variation, instead of temperature change. It is noted that the pressure distribution in Fig. 3(c) calculated through Eq. (1) may not be accurate for confined fluids. A more accurate approach can be found in Ref. [16].

To confirm the flow, the average velocity field is obtained for the case of $\varepsilon_{\mathrm{fw}(L)}=10 \mathrm{~K}$ and $T_{H}=600 \mathrm{~K}$, as illustrated in Fig. 4(a). It is seen that the fluid has a general motion in the negative $x$ direction. The flow fields in the low and high surface energy areas are quite different. In the low surface energy part, the velocity in the center area is larger than that around the wall. In the right half of the flow field, the velocity near the wall at low temperature is almost

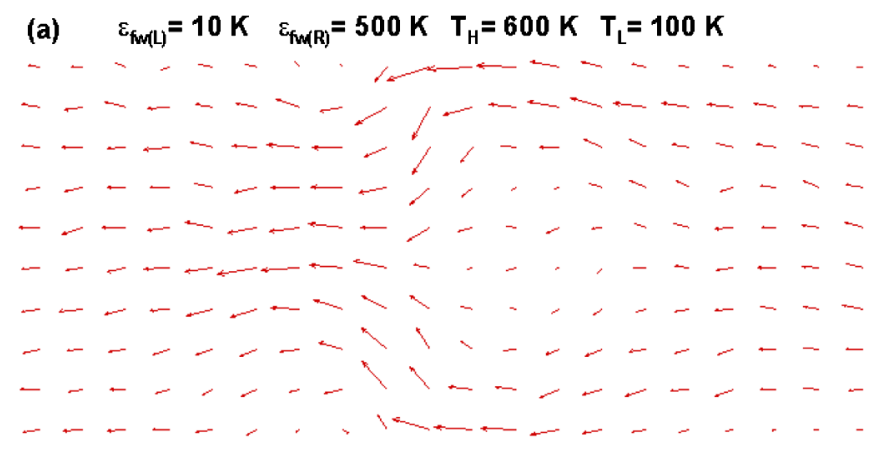

(b) $\varepsilon_{\mathrm{fw}(\mathrm{L})}=200 \mathrm{~K} \quad \varepsilon_{\mathrm{fw}(\mathrm{R})}=500 \mathrm{~K} \quad \mathrm{~T}_{\mathrm{H}}=600 \mathrm{~K} \quad \mathrm{~T}_{\mathrm{L}}=100 \mathrm{~K}$

FIG. 4 (color online). The velocity field in a $4 \mathrm{~nm}$ channel with $T_{H}=600$ and $T_{L}=100 \mathrm{~K}$. (a) $\varepsilon_{\mathrm{fw}(L)}=10$ and $\varepsilon_{\mathrm{fw}(R)}=500 \mathrm{~K}$. (b) $\varepsilon_{\mathrm{fw}(L)}=200$ and $\varepsilon_{\mathrm{fw}(R)}=500 \mathrm{~K}$.

zero because the fluid molecules are adsorbed by the wall. The largest velocity takes place in the area of high temperature around the wall, where fluid molecules have high kinetic energy and are free to move.

Based on the above analysis, to guarantee the flow in the channel under a symmetric temperature gradient, the configuration of the surface energy has to be appropriate such that the fluid adsorption occurs only in one half of the channel. Fluid adsorption on solid surfaces is generally determined by the ratio of fluid-wall binding energy to temperature, $\varepsilon_{\mathrm{fw}} / k T$ [17-22]. This ratio expresses the competition between the fluid-wall binding strength and the kinetic energy of the fluid. Usually fluid adsorption tends to occur when $\varepsilon_{\mathrm{fw}} / k T>1$. Hence, for the given temperature gradients in Fig. 4(a), to guarantee a flow, $\varepsilon_{\mathrm{fw}}>100 \mathrm{~K}$ is required for fluid adsorption in one half of the channel and $\varepsilon_{\mathrm{fw}}<100 \mathrm{~K}$ has to be satisfied in the other half to prevent fluid adsorption. In Fig. 4(a), $\varepsilon_{\mathrm{fw}(L)}=10$ and $\varepsilon_{\mathrm{fw}(R)}=500 \mathrm{~K}$, which satisfy the two conditions, and therefore a clear fluid motion is observed. If one of the conditions is not satisfied, for example, if $\varepsilon_{\mathrm{fw}(L)}$ in the case of Fig. 4(a) is changed from 10 to $200 \mathrm{~K}$, which is about to cause fluid adsorption, the fluid-wall interaction in both the left and right half of the channel becomes similar, as confirmed by the velocity distribution in Fig. 4(b). In this case, the distinct mechanisms required for the flow are not ensured and the fluid motion turns out to be unobservable (Fig. 2). 


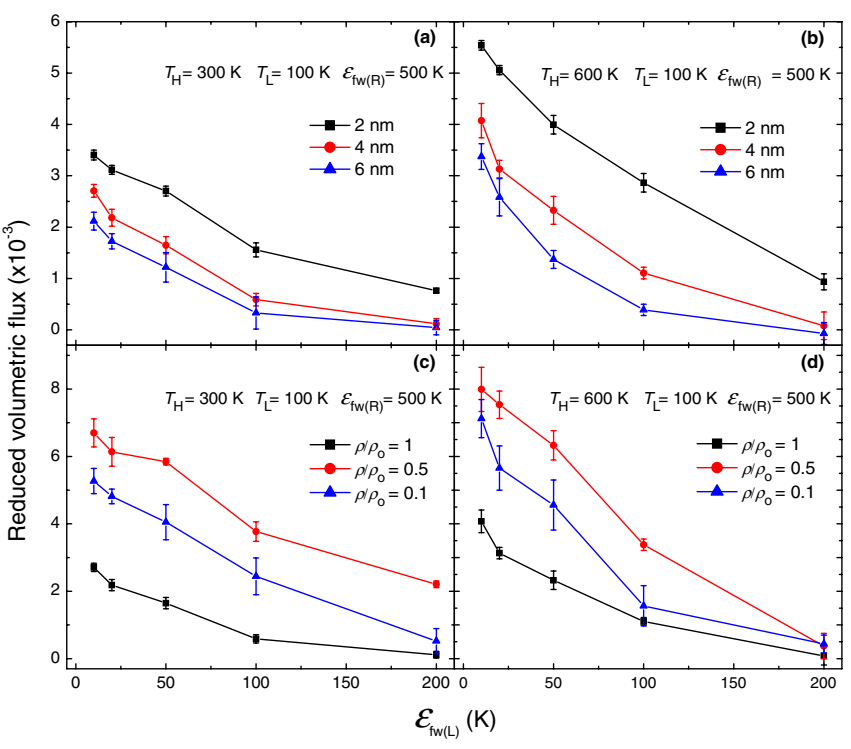

FIG. 5 (color online). The reduced volumetric flux as a function of $\varepsilon_{\mathrm{fw}(L)}$ in 2, 4, and $6 \mathrm{~nm}$ channels (a),(b) and for different fluid densities (c),(d) with $\varepsilon_{\mathrm{fw}(R)}=500 \mathrm{~K}$ and $T_{L}=100 \mathrm{~K}$. In (c) and (d), the channel width is $4 \mathrm{~nm}$.

Since the flow in the channel is caused by the coupling effect of temperature gradient and appropriate surface energy configuration, the channel size must be an important parameter that affects the flow. Other than the $4 \mathrm{~nm}$ channel in Fig. 2, we also performed simulations in 2 and $6 \mathrm{~nm}$ channels. The flux for the same fluid in these channels is depicted in Fig. 5. In Figs. 5(a) and 5(b), $T_{H}=300$ and $600 \mathrm{~K}$, respectively, and $\varepsilon_{\mathrm{fw}(L)}$ is varied from 10 to $200 \mathrm{~K}$. The other conditions are the same as that in Fig. 2. It is seen that the flux increases with decreasing channel size. This is reasonable because the surface effect grows strong as channel size decreases. Furthermore, the effect of fluid density $\rho$ on the flux is also examined. Figures 5(c) and 5(d) show the flux for different values of density ratio $\rho / \rho_{0}$. There seems to be a maximum value in the flux as the density is varied. This might be due to the change in the competition between fluid-wall and fluid-fluid interactions as the mean distance among the fluid molecules and consequently the intermolecular interaction are changed [12]. Figure 5 indicates that the relationship between the flux and the parameters, i.e., channel size, temperature gradient, surface energy, and fluid density, is nonlinear. There may exist an optimal configuration, for which the flux is the maximum. This will be explored in future work.

It is worth mentioning that the proposed system could be used for chip-level cooling. The intrinsic heat generated by the chip provides a symmetric temperature gradient, which can be employed to circulate liquids through channels. Compared with the conventional cooling techniques, the composite nanochannel system is energy friendly since it does not require extra pumps. This is in favor of the miniaturization and integration of electronic devices.

In summary, we have shown that fluids can be pumped by a symmetric temperature gradient in composite nanochannels, which have heterogeneous surface energies. The fluid moves from high to low temperature in the low surface energy part, where the pressure difference is mainly caused by the temperature variation, and from low to high temperature in the high surface energy part, where the pressure change is largely resulted from the density gradient.

This work was supported in part by the Direct Allocation Grant No. DAG07/08.EG02 and School Initiative Grant No. SBI07/08.EG01.

*mezli@ust.hk

[1] T. M. Squires and S. R. Quake, Rev. Mod. Phys. 77, 977 (2005).

[2] T. A. J. Duke and R. H. Austin, Phys. Rev. Lett. 80, 1552 (1998).

[3] S. Ghosh, A. K. Sood, and N. Kumar, Science 299, 1042 (2003).

[4] S. V. Garimella, Microelectron. J. 37, 1165 (2006).

[5] X. Gong, J. Li, H. J. Lu, R. Wan, J. Li, J. Hu, and H. Fang, Nature Nanotech. 2, 709 (2007).

[6] S. Pennathur and J. G. Santiago, Anal. Chem. 77, 6782 (2005).

[7] M. Rauscher, S. Dietrich, and J. Koplik, Phys. Rev. Lett. 98, 224504 (2007).

[8] Z. Guttenberg, A. Rathgeber, S. Keller, J. O. Rädler, A. Wixforth, M. Kostur, M. Schindler, and P. Talkner, Phys. Rev. E 70, 056311 (2004).

[9] M. K. Chaudhury and G. M. Whitesides, Science 256, 1539 (1992).

[10] J. B. Brzoska, F. Brochard-Wyart, and F. Rondelez, Langmuir 9, 2220 (1993).

[11] A. A. Darhuber and S. M. Troian, Annu. Rev. Fluid Mech. 37, 425 (2005).

[12] C. Liu and Z. G. Li, Phys. Rev. E 80, 036302 (2009).

[13] K. P. Travis, B. D. Todd, and D. J. Evans, Phys. Rev. E 55, 4288 (1997).

[14] M. P. Allen and D. J. Tildesley, Computer Simulation of Liquids (Oxford, New York, 1987).

[15] Z. G. Li, Phys. Rev. E 80, 061204 (2009).

[16] B. D. Todd, D. J. Evans, and P. J. Daivis, Phys. Rev. E 52, 1627 (1995).

[17] Z. G. Li and H. Wang, Phys. Rev. Lett. 95, 014502 (2005).

[18] R. S. Voronov, D. V. Papavassiliou, and L. L. Lee, J. Chem. Phys. 124, 204701 (2006).

[19] U. Heinbuch and J. Fischer, Phys. Rev. A 40, 1144 (1989).

[20] Z. G. Li and L. Hong, J. Chem. Phys. 127, 074706 (2007).

[21] P. A. Thompson and M. O. Robbins, Phys. Rev. A 41, 6830 (1990).

[22] M. Cieplak, J. Koplik, and J. R. Banavar, Phys. Rev. Lett. 86, 803 (2001). 